\title{
Improve the Efficiency of Scintillation Detectors Using Reflectors Based on Photonic Crystals Arrays
}

\author{
M. F. Eissa, Arafa H. Aly ${ }^{*}$ \\ Physics Department, Faculty of Science, Beni-Suef University, Beni-Suef, Egypt. \\ Email: ${ }^{*}$ arafaaly@aucegyypt.edu
}

Received December $3^{\text {rd }}$, 2013; revised December $29^{\text {th }}$, 2013; accepted January $19^{\text {th }}$, 2014

Copyright (C 2014 M. F. Eissa, Arafa H. Aly. This is an open access article distributed under the Creative Commons Attribution License, which permits unrestricted use, distribution, and reproduction in any medium, provided the original work is properly cited. In accordance of the Creative Commons Attribution License all Copyrights @ 2014 are reserved for SCIRP and the owner of the intellectual property M. F. Eissa, Arafa H. Aly. All Copyright (C) 2014 are guarded by law and by SCIRP as a guardian.

\begin{abstract}
In the present work, we designed the new type of photonic crystals (PCs) as reflectors. Reflections from single layer of $\mathrm{Al}_{2} \mathrm{O}_{3} / \mathrm{MgO} \mathrm{PC}$ help us in recapturing the light that does escape from the scintillation surface. Photonic crystals in one dimension array of $\mathrm{Al}_{2} \mathrm{O}_{3}$ and $\mathrm{MgO}$ with silver at periodicities $N=1,2$ and 3 were used as a reflector around the surface of the scintillation volume. Scintillation detectors are widely used in nuclear medicine. The efficiency is an important parameter for characterizing the capability of the detectors. The counting efficiency of the detectors depends on the light emission induced by radiation. The light then was converted by the photomultiplier tube into electrical pulses. The efficiency may increase by an amount of $1.64 \%$ if $\mathrm{MgO}$-Ag photonic crystals are used at periodicity $N=1$ as a reflector.
\end{abstract}

\section{KEYWORDS}

\section{TMM; Photonic Crystals; Scintillation Detectors; Radiation; Photonic Band Gap}

\section{Introduction}

The most common scintillator used in nuclear medicine is the NaI (Tl) (thallium-activated sodium iodide) because of its desirable properties. It has excellent absorbance light yield, very low self absorption of scintillation light, easy availability and low production cost $[1,2]$. Scintillation detectors convert the radiation quanta into detectable light. The light is converted into an electrical pulse by photomultiplier tube. The emission spectra of the light produced by $\mathrm{NaI}(\mathrm{Tl})$-crystal are in the wavelength range of $320-550 \mathrm{~nm}$. The wavelength of light has maximum emission at $415 \mathrm{~nm}$ [1].

The efficiency and energy resolution will be helpful in categorizing detector applications. The light collection conditions affect the efficiency of scintillation detectors. Because the scintillation light is emitted in all directions, only a limited fraction can travel directly to the surface at the photomultiplier [1]. To recapture the light that does escape from the surface, the scintillator is normally surrounded by a reflector at all surfaces except that at which

${ }^{*}$ Corresponding author. the photomultiplier tube is mounted. The fraction of reflected light depends on the angle of incidence compared with the critical angle. Better results are obtained with a diffuse reflector such as $\mathrm{MgO}$ or $\mathrm{Al}_{2} \mathrm{O}_{3}$ [1].

Photonic crystals (PCs) are important for a wide range of applications, ranging from basic science to engineering [3-8]. PCs are periodic multilayer structures that possess photonic band gaps (PBGs) which are formed due to the Bragg-Scattering in the periodic structure.

In this work, we have designed the new type of photonic crystals (PCs) as reflectors. Reflectors from single layer of $\mathrm{Al}_{2} \mathrm{O}_{3} / \mathrm{MgO}$ PC help us in recapturing the light that does escape from the scintillation surface. Also we have made attempt to attain reflector which has high reflectance and doesn't depend on the incident angle. This attempt may improve the light collection conditions and result in improving the detection efficiency of the scintillation detector. The single layers of $\mathrm{Al}_{2} \mathrm{O}_{3}$ and $\mathrm{MgO}$ were replaced by a photonic crystal in one dimension array of $\mathrm{Al}_{2} \mathrm{O}_{3}(100 \mathrm{~nm})$ and $\mathrm{MgO}(100 \mathrm{~nm})$ with $8 \mathrm{~nm}$ thickness for silver at periodicities $N=1,2$ and 3 at $\theta=$ $0^{\circ}, 20^{\circ}, 40^{\circ}$ and $60^{\circ}$. 


\section{Theoretical Calculations}

The electromagnetic waves interactions through the multilayer structure is chosen to be along $\mathrm{x}$-direction and this propagation can be described using TMM. This method is based on the analysis of the electric field interaction within the structure along the specified direction in terms of the dynamical and propagating matrices for both TM and TE waves (Figure 1), where the dynamical (D) matrices can be written in the following form $[9,10]$ :

$$
D_{m}=\left(\begin{array}{cc}
1 & 1 \\
n_{j} \cos \theta_{j} & -n_{j} \cos \theta_{j}
\end{array}\right) \text { for TE-Waves }
$$

and,

$$
D_{m}=\left(\begin{array}{cc}
\cos \theta_{j} & \cos \theta_{j} \\
n_{j} & -n_{j}
\end{array}\right) \text { for TM-Waves }
$$

where $j=0,1,2, \cdots$

while the propagation matrices $(P)$ take the form:

$$
P_{m}=\left(\begin{array}{cc}
\cos \phi_{m}+i \sin \phi_{m} & 0 \\
0 & \cos \phi_{m}-i \sin \phi_{m}
\end{array}\right)
$$

where $\phi_{m}=\frac{2 \pi d_{m}}{\lambda} n_{m} \cos \theta_{m}, \quad m=1,2, \cdots$.

Then by using the above expressions for the dynamical and propagating matrices, we can obtain the matrix describing the interaction between the incident electromagnetic waves and the structure as

$$
M(a)=\left(\begin{array}{ll}
m_{11} & m_{12} \\
m_{21} & m_{22}
\end{array}\right)=D_{1} P_{1} D_{1}^{-1} D_{2} P_{2} D_{2}^{-1}
$$

where $M(a)$ is the matrix for one period, Whose elements $m_{11}, m_{12}, m_{21}$ and $m_{22}$ were computed for TEwaves, that can be obtained also for TM-waves using the same analysis, and ( $\left.a=d_{1}+d_{2}\right)$ is the lattice constant.

Finally we can calculate the transmittance and the reflectance using the following expressions:

$$
\begin{gathered}
R=\left|r^{2}\right|, \\
T=\frac{f_{1}}{f_{0}}\left|t^{2}\right|
\end{gathered}
$$

where

$$
f_{0}=\sqrt{\frac{\varepsilon_{0}}{\mu_{0}}} n_{0} \cos \theta_{0} \text { and } f_{1}=\sqrt{\frac{\varepsilon_{0}}{\mu_{0}}} n_{s} \cos \theta_{s}
$$

\section{Results and Discussion}

We have used Essential Macleod software [11]. The Essential Macleod is a comprehensive software package for the design, analysis, manufacture and trouble shooting of thin film optical coatings. The program has a true multiple document interfaces. It can handle coatings from rugates to ultrafast, from single-layer antireflection coatings to demanding color separation beam splitters, providing all that is necessary for their design, analysis, production planning and even reverse engineering of a failed production attempt. A wide range of performance parameters, from straightforward transmittance and reflectance to color coordinates in different color spaces, is built into the software. It can synthesize designs from scratch or refine existing ones, investigate errors and extract optical constants of film materials for use in designs.

The energy lost by the radiation quanta are converted into scintillation photons in scintillation volume. The photons are converted into an electrical pulse by photomultiplier tube. The number of pulses recorded to number of radiation quanta incident on detector is defined as intrinsic efficiency of scintillation detector [1]. Good reflectance for photon by reflector which surrounds the active volume of the scintillation crystal increases the number of pulses recorded. The single layer reflector is similar to the actual fabrication of the Scintillation detectors. The reflector based on photonic crystals is the attempt to improve the light collection conditions.

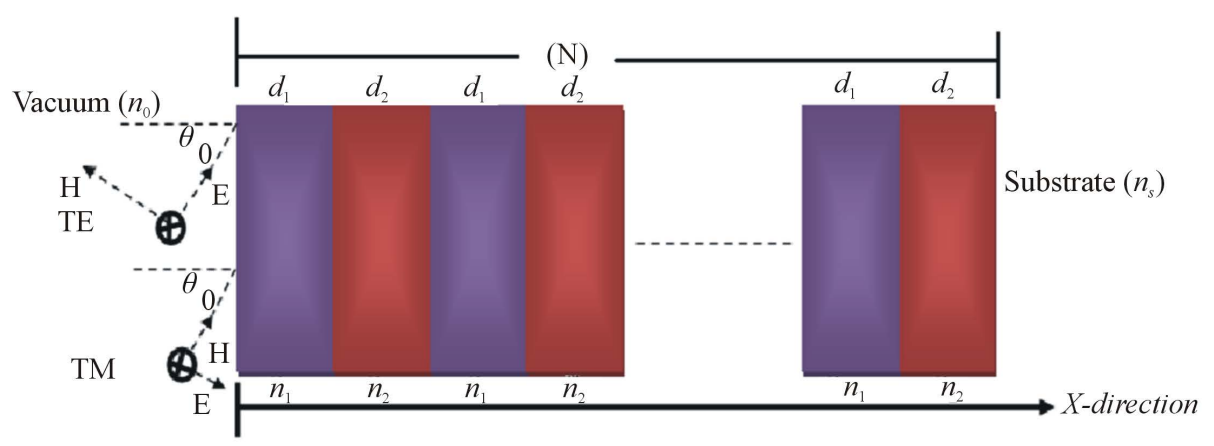

Figure 1. A 1D periodic dielectric structure; the thicknesses of metallic oxide and metallic are denoted by $d_{1}$ and $d_{2}$, respectively, and the corresponding refractive indices are separately indicated by $n_{0}, n_{1}, n_{2}$, and $n_{s}$, where $n_{0}=1$ is taken and $n_{s}$ is the index of substrate layer. 
Therefore, the intrinsic efficiency of the scintillation may improve. The improvement scale for photonic crystal reflector is obtaining reflectance more than its values at single layer. The refractive indices of $\mathrm{NaI}(\mathrm{Tl})$ crystal, $\mathrm{Al}_{2} \mathrm{O}_{3}$ and $\mathrm{MgO}$ were 1.85, 1.772 and 1.744, respectively. Accordingly, the critical angle (total internal reflection occurs) for the wavelength of maximum emission for $\mathrm{NaI}(\mathrm{Tl})(415 \mathrm{~nm})$ reflected at the surface of $\mathrm{Al}_{2} \mathrm{O}_{3} / \mathrm{MgO}$ was $73.30^{\circ} / 70.51^{\circ}$.

The variations of the reflectance with wavelengths (320 - $560 \mathrm{~nm}$ ) for $\mathrm{Al}_{2} \mathrm{O}_{3}$ and $\mathrm{MgO}$ single layer as reflectors with $100 \mathrm{~nm}$ thickness were displayed in Figure 2. The efficiency improvement ratio $(\varepsilon)$ due to using $\mathrm{MgO}$ reflector in the whole wavelength range can be calculated as:

$$
\varepsilon=\left|\frac{A_{\mathrm{Al}_{2} \mathrm{O}_{3}}-A_{\mathrm{MgO}}}{A_{\mathrm{Al}_{2} \mathrm{O}_{3}}}\right| \times 100 \%
$$

where, $A_{\mathrm{Al}_{2} \mathrm{O}_{3}}$ is the area under the $\mathrm{Al}_{2} \mathrm{O}_{3}$ single layer reflectance curve and $A_{\mathrm{MgO}}$ is the area under the $\mathrm{MgO}$ single layer reflectance curve. It is observed from Figure 2 that there is a little difference in the efficiency ratio of the scintillation detector $(0.26 \%)$ as a result of using $\mathrm{MgO}$ reflector. This means that the two reflectors $\left(\mathrm{Al}_{2} \mathrm{O}_{3}\right.$ and $\mathrm{MgO}$ ) give about the same amount of light to the photomultiplier tube in the detection configuration.

Figures 3-6 show the variations of the reflectance with wavelengths $(320-560 \mathrm{~nm})$ for $\mathrm{Al}_{2} \mathrm{O}_{3}$ single layer reflector (100 nm thickness) and photonic crystals (PCs) in one dimension array of $\mathrm{Al}_{2} \mathrm{O}_{3}$ with $8 \mathrm{~nm}$ thickness for silver with periodicities $N=1,2$ and 3 at $\theta=0^{\circ}, 20^{\circ}, 40^{\circ}$ and $60^{\circ}$. The medium and substrate were scintillation volume and aluminum which used as housing for the detector. The improvement ratio of intrinsic efficiency $\left(\varepsilon_{1}\right)$ can be calculated in the wavelengths $(320-560 \mathrm{~nm})$ as:

$$
\varepsilon_{1}=\left|\frac{A_{s}-A_{\mathrm{PCS}}}{A_{\mathrm{s}}}\right| \times 100 \%
$$

where, $A_{\mathrm{pcs}}$ is the area under the photonic reflectance curve and $A_{\mathrm{s}}$ is the area under the single layer reflectance curve. The $\varepsilon_{1}$ for the single layer has no significant change when replaced by photonic crystals array at $N=1$. The arrays at $N=2$ and 3 show decreasing in $\varepsilon_{1}$-value. The $\mathrm{Al}_{2} \mathrm{O}_{3}-\mathrm{Ag}$ photonic crystals reflectors with $N>3$ have bad results compared with its single layer.

Figures 7-10 show the variations of the reflectance with wavelengths (320 - $560 \mathrm{~nm}$ ). This study was done for MgO single layer reflector (100 nm thickness) and photonic crystals in one dimension array of MgO (100 $\mathrm{nm}$ ) with $8 \mathrm{~nm}$ thickness for silver at periodicities $N=1$, 2 and 3 at $\theta=0^{\circ}, 20^{\circ}, 40^{\circ}$ and $60^{\circ}$. The MgO-Ag photonic crystals reflectors at periodicities higher than

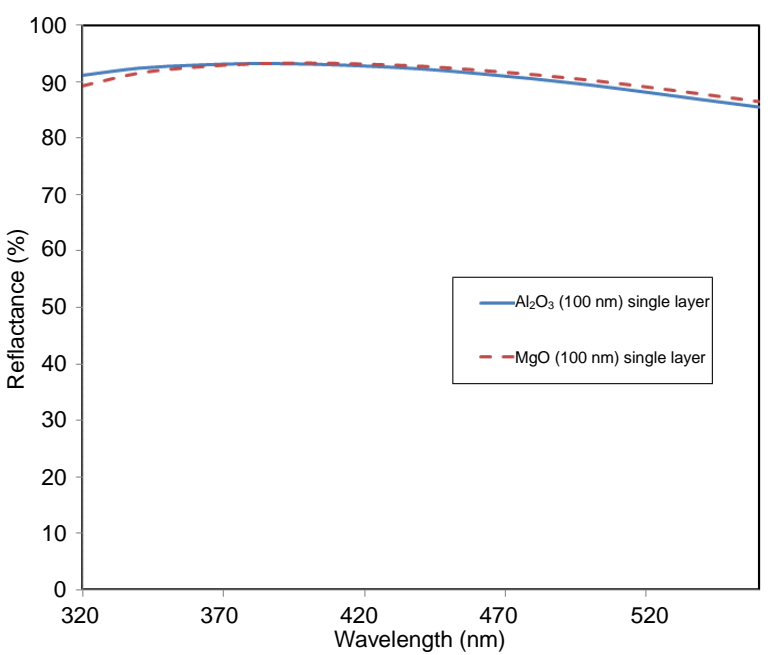

Figure 2. Variations of reflectance with wavelength for $\mathrm{Al}_{2} \mathrm{O}_{3}$ and $\mathrm{MgO}$ with single layer at $\theta=0^{\circ}$.

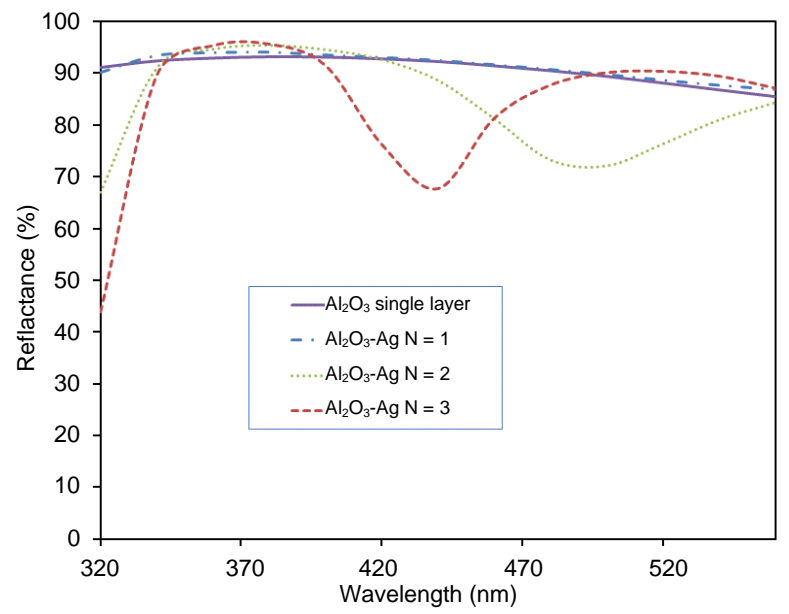

Figure 3. Variations of reflectance with wavelength for single layer of $\mathrm{Al}_{2} \mathrm{O}_{3}$ and $\mathrm{PCs}$ of $\mathrm{Al}_{2} \mathrm{O}_{3}$-Ag at periodicity $N=1$ 3 and $\theta=0^{\circ}$.

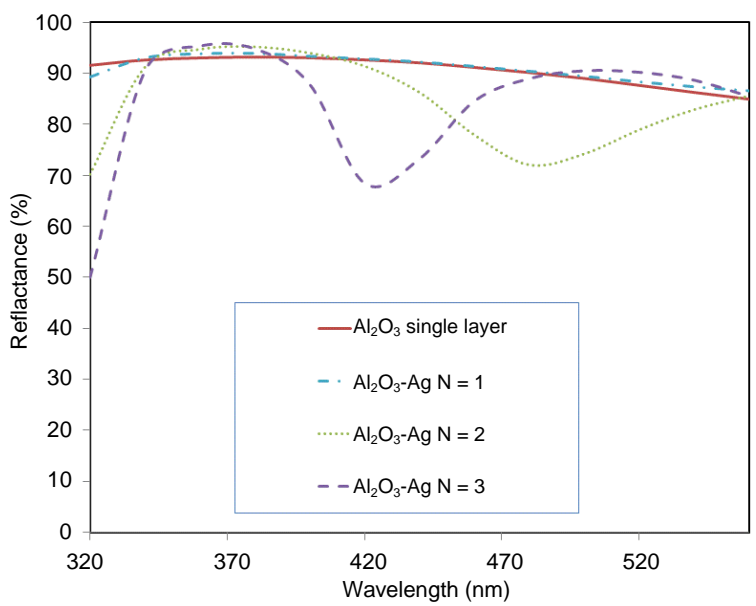

Figure 4. Variations of reflectance with wavelength for single layer of $\mathrm{Al}_{2} \mathrm{O}_{3}$ and $\mathrm{PCs}$ of $\mathrm{Al}_{2} \mathrm{O}_{3}$ - $\mathrm{Ag}$ at periodicity $N=1$ - 




Figure 5. Variations of reflectance with wavelength for single layer of $\mathrm{Al}_{2} \mathrm{O}_{3}$ and $\mathrm{PCs}$ of $\mathrm{Al}_{2} \mathrm{O}_{3}$-Ag at periodicity $N=1$ 3 and $\theta=40^{\circ}$.

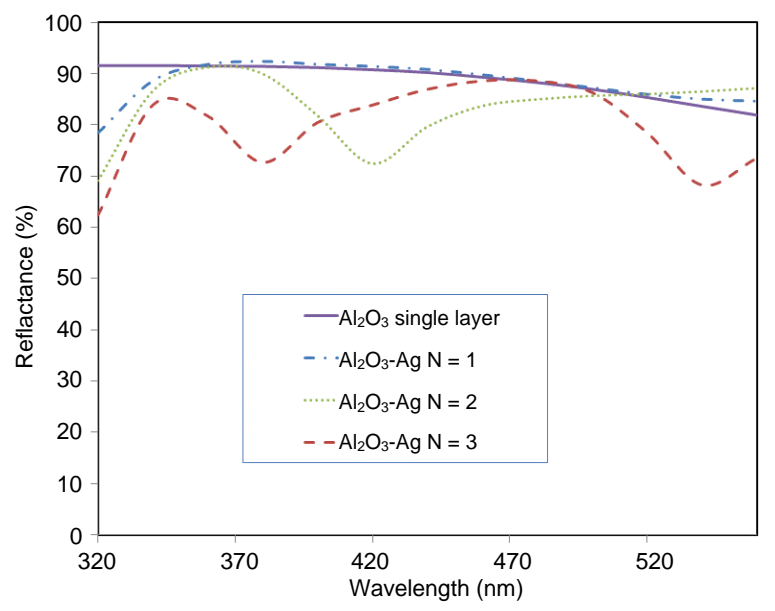

Figure 6. Variations of reflectance with wavelength for single layer of $\mathrm{Al}_{2} \mathrm{O}_{3}$ and $\mathrm{PCs}$ of $\mathrm{Al}_{2} \mathrm{O}_{3}$-Ag at periodicity $N=1$ 3 and $\theta=60^{\circ}$.



Figure 7. Variations of reflectance with wavelength for single layer of $\mathrm{MgO}$ and PCs of $\mathrm{MgO}-\mathrm{Ag}$ at periodicity $N=1$ -
3 and $\theta=0^{\circ}$.

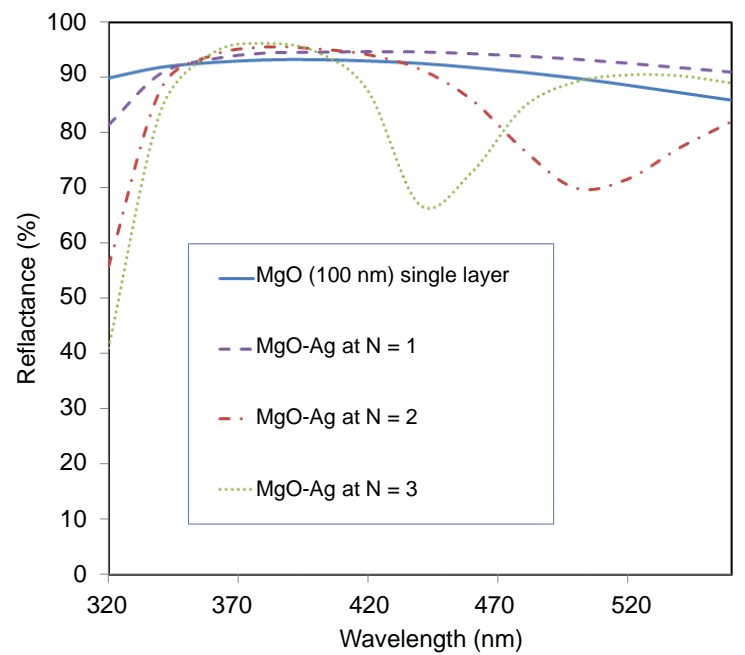

Figure 8. Variations of reflectance with wavelength for single layer of MgO and PCs of MgO-Ag at periodicity $N=1$ 3 and $\theta=20^{\circ}$.



Figure 9. Variations of reflectance with wavelength for single layer of MgO and PCs of MgO-Ag at periodicity $N=1$ 3 and $\theta=40^{\circ}$.



Figure 10. Variations of reflectance with wavelength for single layer of $\mathrm{MgO}$ and PCs of MgO-Ag at periodicity $N=$ 
$1-3$ and $\theta=60^{\circ}$.

$N=3$ have bad results compared with its single layer. The improvement ratio of intrinsic efficiency (Equation (7)) at the single layer has significant change when replaced by photonic crystals array at $N=1$ as in Table 1 . The $\varepsilon_{1}$ approximately have the same value for the different incidence angles. The periodicities at $N=2$ and 3 show less $\varepsilon_{1}$-values.

The variations of reflectance with angle of incidence for single layer and photonic crystals of MgO-Ag with $N$ $=1$ at the characterized wavelength, $415 \mathrm{~nm}$ are represented in Figure 11. It is observed that the reflectance for $\mathrm{MgO}-\mathrm{Ag}$ photonic crystals reflector has higher values and less variations than its single layer.

\section{Conclusion}

The intrinsic efficiency of scintillation detectors may increase by an amount of $1.64 \%$ if we replace the $\mathrm{MgO}$ single crystal reflectors by photonic crystals of MgO-Ag at periodicity $N=1$. The improvement ratio of intrinsic

Table 1. The improvement ratio of intrinsic efficiency replaced MgO single layer by MgO-Ag photonic crystals array at $N=1$ at different incidence angles for the interest wavelengths.

\begin{tabular}{cc}
\hline Incidence angle & $\begin{array}{c}\text { The improvement ratio of } \\
\text { intrinsic efficiency (\%) }\end{array}$ \\
\hline $0^{\circ}$ & 1.67 \\
$20^{\circ}$ & 1.67 \\
$40^{\circ}$ & 1.52 \\
$60^{\circ}$ & 1.69 \\
\hline
\end{tabular}

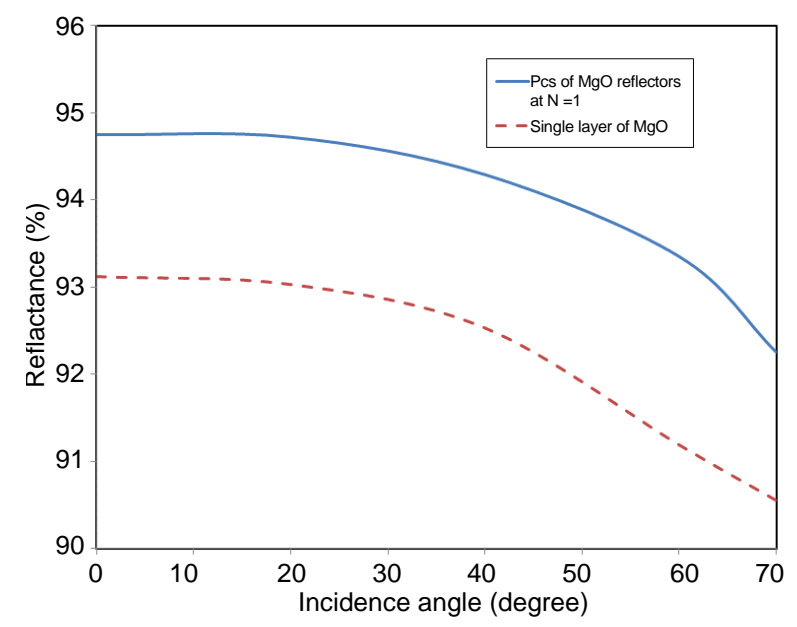

Figure 11. The variations of reflectance with angle of incidence for single layer and PCs of MgO-Ag at the characterized wavelength, $415 \mathrm{~nm}$. efficiency nearly doesn't change if $\mathrm{Al}_{2} \mathrm{O}_{3}$-Ag photonic crystals are used as reflector at periodicity $N=1$. No improvement is found in the improvement ratio for periodicity higher than $N=2$ for both photonic crystal reflectors.

\section{REFERENCES}

[1] G. F. Knoll, "Radiation Detection and Measurement," John Wiley \& Sons, Inc., Hoboken, 2000.

[2] S. N. Ahmed, "Physics and Engineering of Radiation Detection,” Academic Press Inc., Waltham, 2007.

[3] C.-J. Wu, T.-J. Yang, C.-C. Li and P.-Y. Wu, "Investigation of Effective Plasma Frequencies in One-Dimensional Plasma Photonic Crystals," Progress in Electromagnetics Research, Vol. 126, 2012, pp. 521-538. http://dx.doi.org/10.2528/PIER12030505

[4] A. H. Aly, S.-W. Ryu, H.-T. Hsu and C.-J. Wu, “THz Transmittance in One-Dimensional Superconducting Nanomaterial-Dielectric Superlattice," Materials Chemistry and Physics, Vol. 113, No. 1, 2009, pp. 382-384. http://dx.doi.org/10.1016/j.matchemphys.2008.07.123

[5] M. F. Eissa and A. H. Aly, "CR-39 Track Detector as a Photonic Crystal," Journal of Computational and Theoretical Nanoscience, Vol. 10, No. 6, 2013, pp. 1527-1531.

[6] C.-S. Huang, S. George, M. Lu, V. Chaudhery, R. Tan, R. C. Zangar and B. T. Cunningham, "Application of Photonic Crystal Enhanced Fluorescence to Cancer Biomarker Microarrays,” Analytical Chemistry, Vol. 83, No. 4, 2011, pp 1425-1430. http://dx.doi.org/10.1021/ac102989n

[7] A. H Aly, "Electromagnetic Wave Propagation Characteristics in One-Dimensional Photonic Crystals,” In: A. Petrinm, Ed., Handbook of Wave Propagation in Materials for Modern Applications, Chapter 10, Sciyo, 2010, pp. 193-200.

[8] A. Knapitsch, E. Auffray, C. W. Fabjan, J.-L. Leclercq, P. Lecoq, X. Letartre and C. Seassal, "Photonic Crystals: A Novel Approach to Enhance the Light Output of Scintillation Based Detectors," Nuclear Instruments and Methods in Physics Research Section A, Vol. 628, No. 1, 2011, pp. 385-388.

http://dx.doi.org/10.1016/j.nima.2010.07.007

[9] M. Born and E. Wofl, "Principles of Optics," Cambridge University Press, Cambridge, London, 1999. http://dx.doi.org/10.1017/CBO9781139644181

[10] A. H. Aly and H. S. Hanafey, "Polarization Modes Control on the Transmittance Characteristics of One Dimensional Photonic Crystal," Journal of Computational and Theoretical Nanoscience, Vol. 8, No. 10, 2011, pp. 1916-1919. http://dx.doi.org/10.1166/jctn.2011.1901

[11] A. H. Aly, "Personal Communicated with Essential Macleod Software,” Thin Film Center Inc., Tucson, 2008. 